\title{
Massive zero-metal stars: Energy production and mixing
}

\author{
C. W. Straka and W. M. Tscharnuter \\ Institut für Theoretische Astrophysik, Universität Heidelberg, Tiergartenstraße 15, 69121 Heidelberg, Germany
}

Received 21 March 2001 / Accepted 2 April 2001

\begin{abstract}
Time-dependent nuclear network calculations at constant temperature show that for zero-metal stars $\gtrsim 20 M_{\odot}$ (i) $\beta$-decay reactions and (ii) the ${ }^{13} \mathrm{~N}(\mathrm{p}, \gamma){ }^{14} \mathrm{O}$ reaction must be included. It is also shown that the nuclear timescale in these zero-metal stars is shorter than the mixing timescale and therefore the assumption of instantaneous mixing across convective regions is not fulfilled. We conclude that proper modeling of these processes may alter the evolution of massive zero-metal stars.
\end{abstract}

Key words. nuclear reactions - convection - stars: evolution

\section{Introduction}

The evolution of zero-metal stars in the mass range $\sim 20-100 M_{\odot}$, i.e., massive Population III stars (Pop-III for short), has been studied since the pioneering work of Ezer \& Cameron (1971) who followed the evolution of stars in the mass range $5-100 M_{\odot}$ from the pre-main sequence contraction phase until the exhaustion of hydrogen on the main sequence. This opened the field for many studies that were mainly concerned with later evolutionary stages (Cary 1974; Castellani et al. 1983; El Eid et al. 1983; Ober et al. 1983; Klapp 1983, 1984). Motivated by the still ongoing debate about the initial mass function of Pop-III stars, Marigo et al. (2001) present the most comprehensive study of zero-metal evolutionary models $\left(0.7-100 M_{\odot}\right)$ starting from the ZAMS until the AGB in the case of low- and intermediate-mass stars, or to the onset of carbon burning in massive stars.

In earlier studies the authors were forced to make numerous assumptions about equilibria between the chemical species involved. The most recent studies by Marigo et al. (2001), however, incorporate nuclear networks making very few assumptions about equilibria. Nevertheless, even these recent state-of-the-art studies rely on some simplifications that are certainly fulfilled during the evolution of normal stars but are questionable in the case of massive zero-metal stars.

It is the aim of this paper to show that the following two assumptions widely used, namely

1. $\beta$-decay negligible against proton-capture

Send offprint requests to: C. W. Straka, e-mail: cstraka@ita.uni-heidelberg.de
2. instantaneous mixing across convective regions

are not met during the evolution of massive Pop-III stars and that proper modeling may alter the evolution of these objects.

\section{Nuclear network}

It was first noted by Ezer (1961) that Pop-III stars more massive than about $20 M_{\odot}$ are supplied by a different mode of energy generation. Due to the lack of the elements $\mathrm{C}, \mathrm{N}$ and $\mathrm{O}$ the $\mathrm{CNO}$-cycle cannot generate the luminosity needed to halt contraction. This raises the temperature to the point where the $3 \alpha$-reaction produces enough carbon to initiate the CNO-cycle. As a result, the CNO-cycle operates at a much higher temperature, typically at $10^{8} \mathrm{~K}$. Since the energy generation in the CNO-cycle exhibits a strong temperature dependence the central temperatures of the more massive models up to $100 M_{\odot}$ do not exceed $T_{\mathrm{c}}=1.3 \times 10^{8} \mathrm{~K}$ (see e.g., El Eid et al. 1983; Marigo et al. 2001). At these temperatures, the CNO-cycle turnover rate is governed by the beta-decay half-lives (Ezer \& Cameron 1971).

This finding has one important consequence for modeling stellar evolution: one has to include the elements ${ }^{13} \mathrm{~N}$ and ${ }^{15} \mathrm{O}$ (mono-cycle), ${ }^{17} \mathrm{~F}$ (bi-cycle) and ${ }^{18} \mathrm{~F}$ (tri-cycle) explicitely in time-dependent network calculations. In addition, for temperatures exceeding $10^{8} \mathrm{~K}$ the proton capture ${ }^{13} \mathrm{~N}(\mathrm{p}, \gamma){ }^{14} \mathrm{O}$ (followed by ${ }^{14} \mathrm{O}\left(\beta^{+}, \nu\right){ }^{14} \mathrm{~N}$ ) supersedes the ${ }^{13} \mathrm{~N}\left(\beta^{+}, \nu\right){ }^{13} \mathrm{C}$ rate. Contrary to the findings of Klapp (1983) our calculations show a non-negligible effect on the energy generation for $T>10^{8} \mathrm{~K}$.

In order to estimate the described effects, we have performed time-dependent network calculations at constant 

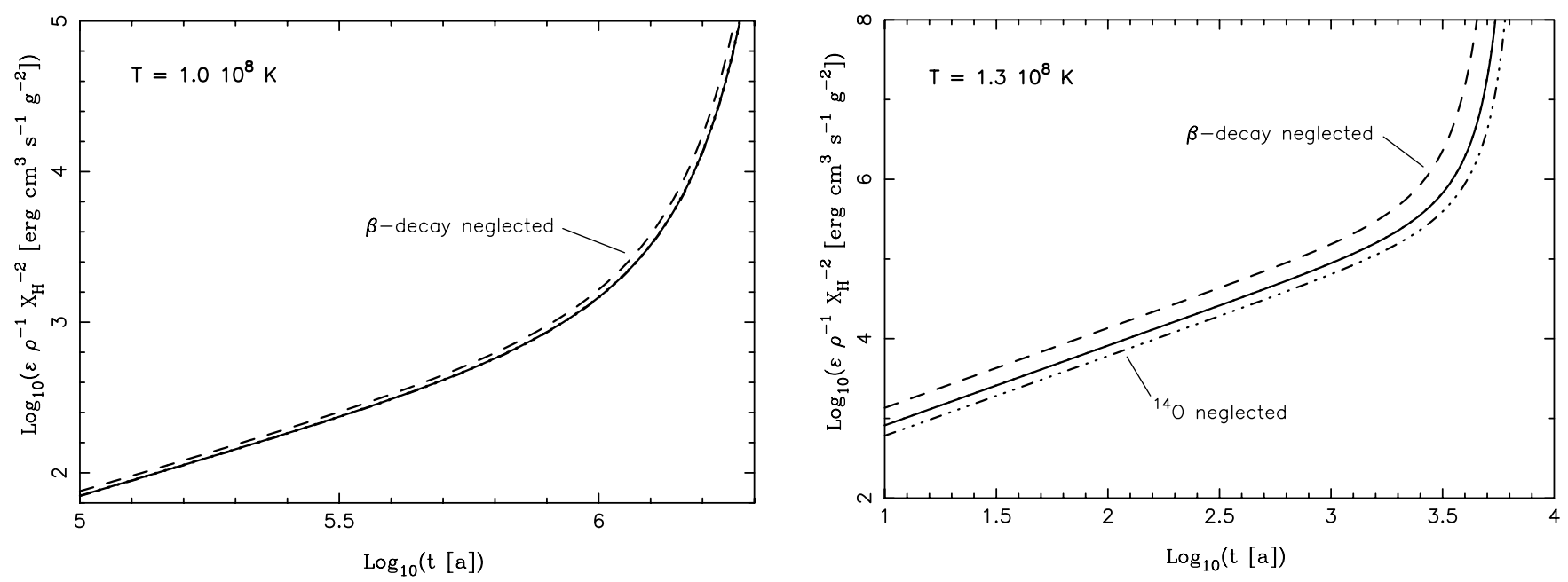

Fig. 1. Energy generation per $\rho X_{\mathrm{H}}^{2}$ at constant temperature for a) $T=1.0 \times 10^{8} \mathrm{~K}$ and $\left.\mathbf{b}\right) T=1.3 \times 10^{8} \mathrm{~K}$. The solid curve is our reference calculation including all 15 chemical species. The dashed curve depicts the model that assumes instantaneous $\beta$ decay and the dotted-dashed curve refers to the model that neglects the reaction ${ }^{13} \mathrm{~N}(\mathrm{p}, \gamma){ }^{14} \mathrm{O}$. Differences between the reference calculation and the calculation neglecting ${ }^{13} \mathrm{~N}(\mathrm{p}, \gamma){ }^{14} \mathrm{O}$ are too small to show up in a).

temperature. Our reference calculation includes 15 chemical species: ${ }^{1} \mathrm{H},{ }^{4} \mathrm{He},{ }^{12} \mathrm{C},{ }^{13} \mathrm{C},{ }^{13} \mathrm{~N},{ }^{14} \mathrm{~N},{ }^{15} \mathrm{~N},{ }^{14} \mathrm{O},{ }^{15} \mathrm{O}$, ${ }^{16} \mathrm{O},{ }^{17} \mathrm{O},{ }^{18} \mathrm{O},{ }^{17} \mathrm{~F},{ }^{18} \mathrm{~F}$ and ${ }^{19} \mathrm{~F}$ with initial abundances of $X_{\mathrm{H}}=0.77, X_{\mathrm{He}}=0.23$ and $X_{\text {other }}=10^{-15}$, and the following reaction chains:

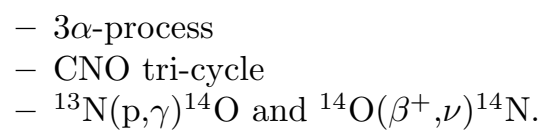

Reaction rates are taken from $N A C R E^{1}$ (see Angulo et al. 1999). The calculations are performed utilizing the DAE solver LIMEX that is maintained by Ehrig \& Nowak (1998).

Our results can be viewed in Fig. 1 where we have plotted the energy generation per $\rho X_{\mathrm{H}}^{2}$ against the time in years for the two cases $T=1.0 \times 10^{8} \mathrm{~K}$ (see Fig. 1a) and $T=1.3 \times 10^{8} \mathrm{~K}$ (see Fig. 1b). Neglecting the beta-decay half-lives (dashed curves) has an impact on the energy generation of $\sim 10 \%$ for $1.0 \times 10^{8} \mathrm{~K}$ and $\sim 65 \%$ for $1.3 \times 10^{8} \mathrm{~K}$. Whereas the proton capture on ${ }^{13} \mathrm{~N}$ alters the energy generation at $1.0 \times 10^{8} \mathrm{~K}$ only slightly $(<1 \%)$, it has a $\sim 30 \%$ effect at $1.3 \times 10^{8} \mathrm{~K}$ (dotted-dashed curve). It is worth noting that the energy generation is only marginally $(<1 \%)$ effected by both the second (named ${ }^{17} \mathrm{ON}$ ) and the third cycle (named ${ }^{19} \mathrm{FO}$ ). These cycles need only be included if one is interested in the relative abundances of the elements $\mathrm{O}$ and $\mathrm{F}$. At constant temperature, the energy production never attains a constant value due to the ongoing feeding of freshly produced carbon via the $3 \alpha$-process.

\section{Mixing}

Massive stars contain large convective cores in which mixing of chemical species occurs due to the turbulent convective motion. In main-sequence stars of Population I or II,

\footnotetext{
${ }^{1}$ http://pntpm.ulb.ac.be/Nacre/
}

this process is very rapid compared to the slow changes of the chemical composition produced by nuclear reactions. Under these circumstances one can safely assume that the composition in a convective region always remains homogeneous, i.e., elements are instantaneously mixed over the convective region. The following arguments are put forward to show that the turn-over time of the CNO-cycle at the high temperatures of massive Pop-III stars can be comparable to the timescale of turbulent mixing - or even shorter.

\subsection{Mixing timescale}

We show that the mixing timescale, $\tau_{\text {conv }}$, is of the order of 10 days in the case of both normal and zero-metal stars. An estimate of this timescale is easily derived from the standard mixing length theory (MLT, Böhm-Vitense 1958) and the formulas we present resemble those given in Kippenhahn \& Weigert (1990, Chap. 7). Let us assume a standard composition of $X_{\mathrm{H}}=0.77$ and $X_{\mathrm{He}}=0.23$, i.e., $\mu=0.58$, and opacity due to electron scattering $\kappa=0.2\left(1+X_{\mathrm{H}}\right)$, i.e., $\kappa=0.35 \mathrm{~cm}^{2} \mathrm{~g}^{-1}$. Even in the case of hot Pop-III stars, neglecting radiation pressure does not change this order of magnitude estimate and it suffices to assume a monoatomic ideal gas: $\delta=1, c_{\mathrm{P}}=5 \mathfrak{R} / 2 \mu$. The mixing timescale is approximately given by the pressure scale height $H_{\mathrm{P}}$ divided by the velocity of convective motion $v_{\text {conv }}$. With this:

$\tau_{\text {conv }} \sim 10^{8}\left(\frac{r}{R_{\odot}}\right)^{2}\left(\frac{M_{\odot}}{m}\right) \sqrt{\frac{T}{10^{8} \mathrm{~K}}}\left(\frac{10^{-4}}{\sqrt{\nabla-\nabla_{\mathrm{e}}}}\right) \mathrm{s}$

where $r, T$ and $m$ are the radius, temperature and mass, respectively, at one particular locus in a star. The quantity $\nabla-\nabla_{\mathrm{e}}$ is the difference between the actual temperature gradient, $\nabla$, and $\nabla_{\mathrm{e}}$ which describes the variation of $T$ in 
a mass element during its motion. Introducing two dimensionless variables:

$$
\begin{aligned}
U \sim & 2 \times 10^{-11}\left(\frac{m}{M_{\odot}}\right)\left(\frac{T}{10^{8} \mathrm{~K}}\right)^{3 / 2} \\
& \times\left(\frac{100 \mathrm{~g} \mathrm{~cm}^{-3}}{\rho}\right)^{2}\left(\frac{R_{\odot}}{r}\right)^{2}
\end{aligned}
$$

$W=\nabla_{\mathrm{rad}}-\nabla_{\mathrm{ad}}$,

and provided that $U \ll\left(\nabla-\nabla_{\mathrm{e}}\right)^{1 / 2} \ll W$ (see below) the cubic equation of MLT can be simplified yielding

$\sqrt{\nabla-\nabla_{\mathrm{e}}} \sim\left(\frac{8}{9} U W\right)^{1 / 3}$

For an estimate of $\tau_{\text {conv }}$ at the center of a star the first two equations can be even further reduced, since $m=$ $4 / 3 \pi \rho_{\mathrm{c}} r^{3}$ :

$$
\begin{aligned}
\tau_{\text {conv }} \sim & 10^{6}\left(\frac{M_{\odot}}{m}\right)^{1 / 3}\left(\frac{100 \mathrm{~g} \mathrm{~cm}^{-3}}{\rho_{\mathrm{c}}}\right)^{2 / 3}\left(\frac{T_{\mathrm{c}}}{10^{8} \mathrm{~K}}\right)^{1 / 2} \\
& \times\left(\frac{7 \times 10^{-4}}{\sqrt{\nabla-\nabla_{\mathrm{e}}}}\right) \mathrm{s}
\end{aligned}
$$

and

$U \sim 4 \times 10^{-10}\left(\frac{m}{M_{\odot}}\right)^{1 / 3}\left(\frac{100 \mathrm{~g} \mathrm{~cm}^{-3}}{\rho_{\mathrm{c}}}\right)^{4 / 3}\left(\frac{T_{\mathrm{c}}}{10^{8} \mathrm{~K}}\right)^{3 / 2}$

For a typical example, consider a $20 M_{\odot}$ Pop-III star. There we have $T_{\mathrm{c}} \sim 10^{8} \mathrm{~K}, \rho_{\mathrm{c}} \sim 100 \mathrm{~g} \mathrm{~cm}^{-3}$ (see e.g., Ezer \& Cameron 1971; Marigo et al. 2001). Let us choose a mass coordinate: $m=1 M_{\odot}$, hence $U \sim 4 \times 10^{-10}$ from Eq. (6). For reasonable values of $W, W \sim 1 \ldots 100$ it follows that $\left(\nabla-\nabla_{\mathrm{e}}\right)^{1 / 2} \sim 7 \times 10^{-4}$. Note that the assumption $U \ll\left(\nabla-\nabla_{\mathrm{e}}\right)^{1 / 2} \ll W$ is fulfilled. With Eq. (5) we finally arrive at

$\tau_{\text {conv }} \sim 10$ days.

For a star containing metals $\left(Z>10^{-10}\right)$ the central temperature is by a factor of three smaller but as normal stars are less compact also the density goes down by a factor of ten. Thus $\tau_{\text {conv }}$ changes approximately by a factor of two. Similar results are obtained at other loci of the convective core and for stellar masses in the range 20-100 $M_{\odot}$.

\subsection{Nuclear timescale}

A characteristic timescale for nuclear reactions in massive stars is the turn-over time of the CNO-cycle. This time is primarily given by the slowest reaction of the cycle which is ${ }^{14} \mathrm{~N}(\mathrm{p}, \gamma){ }^{15} \mathrm{O}$. Even in the high temperature regime $(T=$ $1.3 \times 10^{8} \mathrm{~K}$ ) where proton-captures become comparable to $\beta$-decays this estimate holds within a factor of two. Hence,

$\tau_{\text {nuc }} \sim \tau_{\text {n14pg }}=\frac{A_{\mathrm{H}}}{X_{\mathrm{H}} \rho \lambda_{\mathrm{n} 14 \mathrm{pg}}(T)}$,

where $A_{\mathrm{H}}, X_{\mathrm{H}}$ and $\rho$ are the atomic mass of hydrogen, the mass abundance of hydrogen and the density, respectively (see Clayton 1983). The reaction rate $\lambda_{\mathrm{n} 14 \mathrm{pg}}(T)$

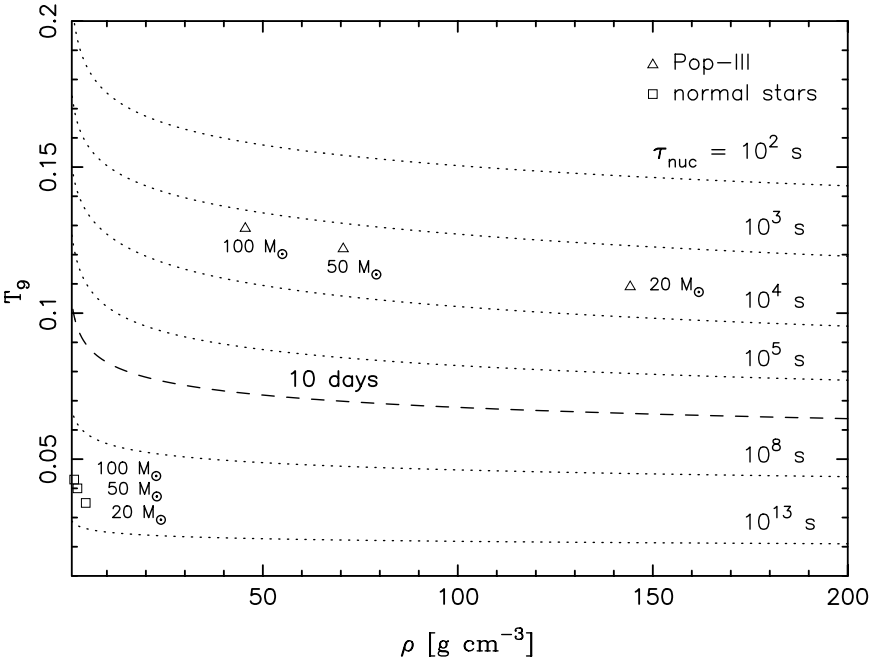

Fig. 2. Lines of constant $\tau_{\text {nuc }}$ at $X_{\mathrm{H}}=0.77$. In the region above the dashed line the mixing time is slow against the nuclear timescale.

measured in $\left[\mathrm{cm}^{3} \mathrm{Mol}^{-1} \mathrm{~s}^{-1}\right]$ is only a function of temperature. Taking this reaction rate again from NACRE (see Angulo et al. 1999):

$$
\begin{aligned}
\lambda_{\mathrm{n} 14 \mathrm{pg}}(T)= & 4.83 \times 10^{7} T_{9}^{-2 / 3} \\
& \times \exp \left(-15.231 T_{9}^{-1 / 3}-\left(\frac{T_{9}}{0.8}\right)^{2}\right) \\
& \times\left(1-2.00 T_{9}+3.41 T_{9}^{2}-2.43 T_{9}^{3}\right) \\
& +2.36 \times 10^{3} T_{9}^{-3 / 2} \exp \left(-3.010 T_{9}^{-1}\right) \\
& +6.72 \times 10^{3} T_{9}^{0.380} \exp \left(-9.530 T_{9}^{-1}\right),
\end{aligned}
$$

the nuclear timescale is easily calculated for a given set of density $\rho$ and temperature $T$. To explore the parameter space we plot lines of constant time $\tau_{\text {nuc }}$ in a $\rho-T$ diagram (see Fig. 2). The dashed line marks the typical timescale of convective mixing. Thus, the region above (below) this line is characterized by slow (fast) mixing compared to the nuclear timescale. The assumption of instantaneous mixing is only justified in the lower region where mixing is fast. It can be nicely seen that all normal stars lie in the lower region (left corner) but that massive Pop-III stars are situated in the upper region where the assumption of instantaneous mixing does not hold.

\section{Conclusions}

Performing time-dependent nuclear network calculations at constant temperature neglecting the $\beta$-decay against proton-capture leads to a considerable error in the energy generation rate. In order to assess the impact of this finding on massive Pop-III stars $\left(\gtrsim 20 M_{\odot}\right)$ detailed stellar modeling is required. In addition, the reaction ${ }^{13} \mathrm{~N}(\mathrm{p}, \gamma){ }^{14} \mathrm{O}$ cannot be omitted for temperatures exceeding $10^{8} \mathrm{~K}$. 
Moreover, the nuclear timescale of massive Pop-III stars can be very short (order of hours) compared to the timescale of convective mixing which is of the order of 10 days. Therefore, instantaneous mixing which is well justified in normal stars may introduce large errors in evolutionary calculations of zero-metal stars.

Contrary to normal stars massive Pop-III stars have not forgotten their nuclear history since equilibrium abundances between chemical species in the CNO-cycle are not attained on the main-sequence. This is due to the continuous production of carbon via the $3 \alpha$-process. Hence, evolutionary calculations must start on the pre-main sequence well before the onset of nuclear reactions.

Acknowledgements. We thank W. J. Duschl for helpful discussions and for improving the manuscript. We are particularly grateful to R. Ehrig for providing and supporting LIMEX. We also thank the referee, A. Weiss, for valuable comments. Part of this work was supported by the German Deutsche Forschungsgemeinschaft, DFG (SFB 439 Galaxies in the Young Universe).

\section{References}

Angulo, C., Arnould, M., Rayet, M., et al. 1999, Nucl. Phys. A, 656, 3

Böhm-Vitense, E. 1958, Z. Astrophys., 46, 108

Cary, N. 1974, Ap\&SS, 31, 3

Castellani, V., Chieffi, A., \& Tornambé, A. 1983, ApJ, 272, 249

Clayton, D. D. 1983, Principles of Stellar Evolution and Nucleosynthesis (The University of Chicago Press, Chicago)

Ehrig, R., \& Nowak, U. 1998, LIMEX Version 4.1A1 (KonradZuse-Zentrum für Informationstechnik, Berlin)

El Eid, M. F., Fricke, K. J., \& Ober, W. W. 1983, A\&A, 119, 54

Ezer, D. 1961, ApJ, 133, 159

Ezer, D., \& Cameron, A. G. W. 1971, Ap\&SS, 14, 399

Kippenhahn, R., \& Weigert, A. 1990, Stellar Structure and Evolution (Springer-Verlag, Berlin Heidelberg)

Klapp, J. 1983, Ap\&SS, 93, 313

Klapp, J. 1984, Ap\&SS, 106, 215

Marigo, P., Girardi, L., Chiosi, C., \& Wood, P. R. 2001, A\&A, 371,152

Ober, W. W., El Eid, M. F., \& Fricke, K. J. 1983, A\&A, 119, 61 